\title{
Pleiotropic effects of obesity-susceptibility loci on metabolic traits: a meta-analysis of up to 37,874 individuals
}

\author{
J. V. van Vliet-Ostaptchouk • M. den Hoed • J. Luan • J. H. Zhao • K. K. Ong • \\ P. J. van der Most • A. Wong • R. Hardy • D. Kuh • M. M. van der Klauw • \\ M. Bruinenberg • K. T. Khaw • B. H. R. Wolffenbuttel • N. J. Wareham • \\ H. Snieder • R. J. F. Loos
}

Received: 17 March 2013 / Accepted: 12 June 2013 / Published online: 5 July 2013

(C) Springer-Verlag Berlin Heidelberg 2013

\begin{abstract}
Aims/hypothesis Genetic pleiotropy may contribute to the clustering of obesity and metabolic conditions. We assessed whether genetic variants that are robustly associated with BMI and waist-to-hip ratio (WHR) also influence metabolic and cardiovascular traits, independently of obesity-related traits, in meta-analyses of up to 37,874 individuals from six European population-based studies.

Methods We examined associations of $32 \mathrm{BMI}$ and $14 \mathrm{WHR}$ loci, individually and combined in two genetic predisposition scores (GPSs), with glycaemic traits, blood lipids and BP, with and without adjusting for BMI and/or WHR.

Results We observed significant associations of BMIincreasing alleles at five BMI loci with lower levels of $2 \mathrm{~h}$
\end{abstract}

Electronic supplementary material The online version of this article (doi:10.1007/s00125-013-2985-y) contains peer-reviewed but unedited supplementary material, which is available to authorised users.

J. V. van Vliet-Ostaptchouk · M. M. van der Klauw •

B. H. R. Wolffenbuttel

Department of Endocrinology, University of Groningen,

University Medical Center Groningen, Groningen, The Netherlands

J. V. van Vliet-Ostaptchouk $\cdot$ P. J. van der Most $\cdot$ H. Snieder Unit of Genetic Epidemiology and Bioinformatics, Department of Epidemiology, University of Groningen, University Medical Center Groningen, Groningen, The Netherlands

J. V. van Vliet-Ostaptchouk · M. M. van der Klauw •

M. Bruinenberg $\cdot$ B. H. R. Wolffenbuttel

LifeLines Cohort Study, University of Groningen, University

Medical Center Groningen, Groningen, The Netherlands

M. den Hoed · J. Luan • J. H. Zhao • K. K. Ong • N. J. Wareham •

R. J. F. Loos

Medical Research Council Epidemiology Unit,

Institute of Metabolic Science, Addenbrooke's Hospital,

Cambridge, UK glucose ( $R B J$ [also known as DNAJC27], QPTCL: effect sizes -0.068 and $-0.107 \mathrm{SD}$, respectively), HDL-cholesterol (SLC39A8: -0.065 SD, MTCH2: -0.039 SD), and diastolic BP (SLC39A8: -0.069 SD), and higher and lower levels of LDL- and total cholesterol (QPTCL: 0.041 and 0.042 SDs, respectively, FLJ35779 [also known as POC5]: -0.042 and -0.041 SDs, respectively) (all $p<2.4 \times 10^{-4}$ ), independent of BMI. The WHR-increasing alleles at two WHR loci were significantly associated with higher proinsulin (GRB14: $0.069 \mathrm{SD})$ and lower fasting glucose levels (CPEB4: -0.049 $\mathrm{SD}$ ), independent of BMI and WHR. A higher GPS-BMI was associated with lower systolic BP $(-0.005 \mathrm{SD})$, diastolic BP $(-0.006 \mathrm{SD})$ and $2 \mathrm{~h}$ glucose $(-0.013 \mathrm{SD})$, while a higher GPS-WHR was associated with lower HDL-cholesterol

M. den Hoed

Department of Medical Sciences, Molecular Epidemiology and Science for Life Laboratory, Uppsala University, Uppsala, Sweden

A. Wong $\cdot$ R. Hardy $\cdot$ D. Kuh

MRC Unit for Lifelong Health and Ageing, Institute of

Epidemiology and Health Care, University College London,

London, UK

\section{K. T. Khaw}

Department of Public Health and Primary Care,

University of Cambridge, Cambridge, UK

\section{R. J. F. Loos $(\bowtie)$}

Genetics of Obesity and Related Metabolic Traits Program, The Charles Bronfman Institute for Personalized Medicine, The Mindich Child Health and Development Institute, Department of Preventive Medicine, Icahn School of Medicine at Mount Sinai, 1 Gustave L. Levy Place, Box 1003, New York, NY 10029, USA e-mail: ruth.loos@mssm.edu 
$(-0.015 \mathrm{SD})$ and higher triacylglycerol levels $(0.014 \mathrm{SD})$ (all $\left.p<2.9 \times 10^{-3}\right)$, independent of BMI and/or WHR.

Conclusions/interpretation These pleiotropic effects of obesitysusceptibility loci provide novel insights into mechanisms that link obesity with metabolic abnormalities.

Keywords BMI · Genetic $\cdot$ Meta-analysis $\cdot$ Metabolic traits $\cdot$ SNP $\cdot$ Obesity susceptibility $\cdot$ Pleiotropy $\cdot$ WHR

\begin{tabular}{|c|c|}
\hline \multicolumn{2}{|c|}{ Abbreviations } \\
\hline $\mathrm{BF} \%$ & Body-fat percentage \\
\hline EPIC & $\begin{array}{l}\text { European Prospective Investigation } \\
\text { into Cancer and Nutrition }\end{array}$ \\
\hline GPS & Genetic predisposition score \\
\hline GPS-BMI & Genetic predisposition score for BMI \\
\hline GPS-WHR & Genetic predisposition score for WHR \\
\hline GWAS & Genome-wide association study \\
\hline HWE & Hardy-Weinberg equilibrium \\
\hline LD & Linkage disequilibrium \\
\hline MRC & Medical Research Council \\
\hline NSHD & $\begin{array}{l}\text { MRC National Survey of Health } \\
\text { and Development }\end{array}$ \\
\hline QC & Quality control \\
\hline SNP & Single-nucleotide polymorphism \\
\hline WHR & Waist-to-hip-ratio \\
\hline
\end{tabular}

Obesity is a key risk factor for a number of metabolic disorders, including type 2 diabetes, dyslipidaemia and cardiovascular disease, and has been associated with increased mortality [1-6]. Although the exact biological mechanisms linking obesity to these comorbidities are not fully understood, it has been shown that increased adiposity, especially abdominal fat accumulation, over time leads to a cluster of metabolic and cardiovascular abnormalities, including elevated blood glucose, insulin resistance, raised triacylglycerol, lower HDL-cholesterol and high BP [7, 8]. The clustering of obesity with clinical features of metabolic disease might, at least in part, be explained by the presence of shared aetiological factors, such as a set of genetic factors with pleiotropic effects, i.e. genes that affect multiple traits $[9,10]$. Examining the pleiotropic effects of obesity-susceptibility loci may, therefore, provide novel insights into underlying biological and molecular mechanisms that link obesity with metabolic abnormalities.

Large-scale genome-wide association studies (GWASs) have identified a number of genetic loci that are robustly associated with obesity phenotypes. To date, there are 32 established loci for BMI, a measure of overall obesity [11-16], and 14 loci associated with BMI-adjusted waistto-hip ratio (WHR), representing the risk for increased abdominal adiposity independent of overall obesity, that have been identified in populations of European descent [17, 18].
Seven of the 14 WHR-associated loci show stronger associations in women than in men [17].

In the present study, we examined pleiotropic effects of the currently GWAS-established obesity-susceptibility loci on metabolic and cardiovascular traits. We systematically assessed associations of the 32 BMI-increasing and 14 WHR-increasing alleles, individually and combined, with glucose-related traits, blood lipids and BP. We examined whether the variants' associations with related traits are likely to be mediated by their effects on obesity-related traits by testing associations with and without adjusting for BMI and WHR in meta-analyses of up to 37,874 individuals from six population-based studies of European origin.

\section{Methods}

Study populations

We combined data from six studies comprising data from up to 37,874 individuals of European origin in meta-analyses: the European Prospective Investigation into Cancer and Nutrition (EPIC)-Norfolk Cohort study $(n=22,887)$ [19]; the LifeLines cohort study $(n=7,846)[20]$; the Fenland study, samples $1(n=1,402)$ and $2(n=3,186)[21,22]$; the Medical Research Council (MRC) Ely study $(n=1,602)$ [23]; and the MRC National Survey of Health and Development (NSHD) study $(n=951)$ [24, 25] (electronic supplementary material [ESM] Table 1). This sample represents nondiabetic individuals only, i.e. those with self-reported type 1 or 2 diabetes, individuals using blood-glucoselowering medication and/or individuals with fasting glucose levels $\geq 7 \mathrm{mmol} / \mathrm{l}$ were excluded. All participants underwent an examination for anthropometric and BP measurements, and blood samples were taken for analysis of laboratory markers (see ESM Methods and Results for study-specific details). All participants gave their written informed consent and all study protocols were approved by local institutional review boards.

\section{Genotyping}

Thirty-two single-nucleotide polymorphisms (SNPs), representing the GWAS-established BMI loci [14], and 14 SNPs for the GWAS-established WHR loci [17] (or their proxies with $r^{2}$ for linkage disequilibrium [LD] $\left.>0.7\right)$ were genotyped in all six studies. Genotype data were derived from various genotyping platforms. Detailed information on selected SNPs, genotyping methods and platforms, quality control (QC) and other filters used for each study are described in ESM Tables 2 and 3 and ESM Methods and Results. In short, we excluded low-quality samples and SNPs before imputation in studies with genome-wide data 
using standard QC criteria (call rate $<95 \%$, minor allele frequency $[\mathrm{MAF}]<1 \%$, Hardy-Weinberg equilibrium $[\mathrm{HWE}]<10^{-4}$ or HWE $<10^{-6}$ ). The quality-controlled data were used to impute genotypes in LifeLines and Fenland study, sample 1. All SNPs or their proxies met QC criteria (call rate $>95 \%$, blind duplicate concordance $>97 \%$ and genotype distributions in HWE, $p>0.001$ [Bonferroni-corrected $p$ value for the 46 SNPs at level $a=0.05$ ], imputation score $\geq 0.3$ ) (for details see ESM Table 3 and ESM Methods and Results). In the LifeLines study, rs11847697 (BMI-associated locus near PRKD1) and rs6905288 (WHR-associated locus near $V E G F A$ ) were excluded from analyses because of a low imputation score (i.e. $<0.3$ ).

\section{Statistical analyses}

Study-specific analysis In studies with directly genotyped data, SNPs were coded as 0,1 or 2 according to the number of BMI- or WHR-increasing alleles for each SNP, defined on the basis of the results from the most recent and largest GWAS for BMI and WHR [14, 17]. In the studies with imputed genotype data, genotypes were coded as dosages with fractional values ranging between 0 and 2, corresponding to the estimated number of copies of the effect allele for a given SNP. We used linear regression to examine the associations of each SNP with BMI or WHR, body-fat percentage (BF\%), leptin, fasting glucose, $2 \mathrm{~h}$ glucose, fasting insulin, proinsulin and $\mathrm{HbA}_{1 \mathrm{c}}$ levels, adjusting for age in men and women separately. Associations with blood-lipid levels were additionally adjusted for the use of lipid-lowering medication and associations with systolic and diastolic BP for the use of BP-lowering medication. As WHR loci were identified after adjusting for BMI [17, 18], we additionally adjusted association analyses of the WHR SNPs for BMI. As distributions for fasting insulin and proinsulin were right-skewed, we logtransformed (natural $\log$ ) values to approximate a normal distribution prior to the analyses.

To examine whether associations between the obesitysusceptibility loci and metabolic and cardiovascular traits were independent of their effects on adiposity level (referred to as 'pleiotropic effects' further in the text), all association analyses were performed with and without additionally adjusting for BMI or WHR (ESM Fig. 1), as appropriate. All analyses were repeated after inverse normal transformation of traits to a mean of 0 and a SD of 1 to allow comparing effect sizes across traits. Phenotypic correlations of BMI/WHR with metabolic traits were calculated using Pearson correlation coefficients.

To assess the combined effect of the obesity-susceptibility loci, we calculated genetic predisposition scores (GPS) for BMI (GPS-BMI) and WHR (GPS-WHR) loci separately by summing the number of effect alleles carried by each individual. Individuals with missing genotypes for more than
$10 \%$ of the loci (i.e. more than three BMI SNPs or more than one WHR SNP) were excluded from the GPS analyses. As a result, 7,023 individuals were excluded from the GPS-BMI analyses and 3,557 individuals from the GPS-WHR analyses (i.e. $18.5 \%$ and $9.4 \%$ of the total available sample used for a single-SNP analysis, respectively), resulting in a total sample of 30,790 and 34,179 for the GPS-BMI and GPS-WHR analyses, respectively. In individuals with fewer than $10 \%$ of the genotypes missing, the missing genotypes were substituted with the average allele count of the respective SNP in each individual study. In the LifeLines study, in which information on two SNPs was missing, genotypes for rs11847697 (BMI locus near PRKD1) and rs6905288 (WHR locus near VEGFA) were replaced with the average allele count of the respective SNPs in Fenland study sample 1, because of similarities in study population and genotyping platform used.

In EPIC-Norfolk, the largest study included in our metaanalyses, the GPS-BMI and GPS-WHR were normally distributed with a mean $( \pm \mathrm{SD})$ of $27.6( \pm 3.4)$ BMI- and 14.1 $( \pm 2.4)$ WHR-increasing alleles. Distributions were similar in the other studies.

Association analyses for the GPS were performed in men and women combined, in the same way as the single-SNP analyses, and were additionally adjusted for sex. Because of the reported sexual dimorphism of WHR [17], the analysis of the GPS-WHR association with WHR was also performed for men and women separately. To test for sex differences, a $\mathrm{SNP} \times$ sex or GPS-WHR $\times$ sex interaction term was added to the model when appropriate. Statistical analyses were performed using SAS, version 9.2, for Windows (SAS Institute, Cary, NC, USA).

Meta-analysis Summary statistics of individual studies ( $\beta$ coefficients and standard errors) were meta-analysed using the inverse-variance method of METAL software (www.sph.umich.edu/csg/abecasis/Metal/) [26].

The statistical significance of the confirmatory associations between single SNPs and BMI and WHR was defined as a nominal significance level of $p<0.05$. For the exploratory pleiotropic association, we accounted for the number of independent tests using a Bonferroni correction. As such, $p<2.72 \times 10^{-4}(\alpha=0.05 /[46 \mathrm{SNPs} \times$ four trait clusters] $)$ and $p<6.25 \times 10^{-3}(\alpha=0.05 /[$ two genetic predisposition scores $\times$ four trait clusters]) were considered statistically significant in the single SNP and the GPS analysis, respectively. The four trait clusters were defined as: (1) adiposityrelated traits (BF\%, leptin levels); (2) glucose- and insulinrelated traits (fasting glucose, $2 \mathrm{~h}$ glucose, $\mathrm{HbA}_{1 \mathrm{c}}$, insulin and proinsulin levels); (3) blood lipids (HDL-cholesterol, LDL-cholesterol, total cholesterol and triacylglycerol levels); and (4) haemodynamic traits (diastolic and systolic BP).

Forest plots of the significant associations were generated using Stata software (version 11; StataCorp, College Station, 
TX, USA). Heterogeneity across studies was estimated using the $Q$ statistic for heterogeneity implemented in METAL [26] and quantified by the $I^{2}$ index.

Statistical power We used Quanto software (http://hydra. usc.edu/gxe) to calculate the statistical power of our study to detect effect sizes of at least the same magnitude as those reported in the discovery studies $[14,17]$. We used allele frequencies of BMI- and WHR-related variants and effect sizes reported in the most recent and largest GWASs for BMI and WHR, respectively [14, 17]. For each BMI SNP, our study had a statistical power from $35.5 \%$ to $100 \%$ to detect effect sizes with a range from 0.06 to $0.39 \mathrm{~kg} / \mathrm{m}^{2}$ (or larger) at a nominal significance level of 0.05 , given a sample size ranging from 27,000 to 35,000 individuals (depending on outcome and on individual SNP) and effect allele frequencies ranging from $10 \%$ to $90 \%$ (ESM Table 4). More specifically, we had $80 \%$ power to detect previously reported associations for 20 of the 32 BMI loci. For each WHR SNP, with effect allele frequencies ranging from $20 \%$ to $70 \%$, our study had a statistical power ranging from $67.9 \%$ to $100 \%$ to detect effect sizes ranging from 0.02 to 0.04 $\mathrm{SD} /$ allele at a nominal significance level of $p=0.05$ (ESM Table 4). As such, we had $80 \%$ power to detect previously reported associations for 12 of the 14 WHR loci. In addition, our study had sufficient power (i.e. $>80 \%$ ) to detect effect sizes as low as $0.05 \mathrm{SD} /$ allele for associations of individual BMI and WHR SNPs with metabolic traits (ESM Fig. 2).

\section{Results}

The association of the obesity-susceptibility loci with adiposity-related traits

Associations with BMI were directionally consistent with results from the discovery studies [11-18] for all BMIassociated loci except the MTIF3 locus (rs4771122) (ESM Table 5). Associations with BMI reached nominal significance for $23(72 \%)$ of the 32 BMI SNPs $(p<0.05)$, which is in close agreement with the number of loci expected based on our power calculation. In addition, the BMI-increasing alleles of 12 BMI SNPs were nominally significantly associated with higher BF\% and those of five BMI SNPs with higher circulating leptin levels (ESM Table 5). Furthermore, each additional BMI-increasing allele in the GPS-BMI was associated with a $0.028 \mathrm{SD} /$ allele (Fig. 1a) or $0.12 \mathrm{~kg} / \mathrm{m}^{-2}$ allele $^{-1}$ higher BMI, which is equivalent to a $347 \mathrm{~g}$ heavier body weight in adults of $170 \mathrm{~cm}$ in height $\left(p<1.1 \times 10^{-61}\right)$ (ESM Table 5). The GPS-BMI was also significantly associated with a $0.016 \mathrm{SD} /$ allele higher $\mathrm{BF} \%\left(p=1.7 \times 10^{-19}\right)$ and $0.022 \mathrm{SD} /$ allele higher leptin levels $\left(p=3.3 \times 10^{-4}\right)$ (Table 1). Of note, the effects sizes of the GPS-BMI were smaller for $\mathrm{BF} \%$ than for BMI, very likely because BMI is only a proxy for overall adiposity that represents a composite trait of both fat and lean mass.

Associations with WHR adjusted for BMI were directionally consistent with findings of the discovery study for all 14 loci [17], and reached nominal significance for 12 loci (86\%) (ESM Table 6$)$. The effects were significantly $\left(p_{\text {interaction }}<2.4 \times 10^{-3}\right)$ larger in women than in men for five of the seven WHR loci for which a sexual dimorphism was observed previously [17]. We did not confirm sex differences for the HOXC13 (rs1443512) or RSPO3 (rs9491696) loci (effect size 0.042 in women vs 0.026 $\mathrm{SD}$ in men, $p_{\text {interaction }}=0.37$; effect size $0.041 \mathrm{SD}$ vs $0.035 \mathrm{SD}$, $p_{\text {interaction }}=0.37$, respectively). Yet, we did observe a stronger association of $L Y 86$ (rs1294421) with WHR in women than in men (effect size $0.039 \mathrm{SD}$ vs $0.011 \mathrm{SD}, p_{\text {interaction }}=0.05$ ). SNPs for three WHR loci were significantly associated with BF\% but, interestingly, the WHR-increasing alleles for two of these (LYPLAL1 and GRB14) were associated with lower BF\%. No association of the WHR loci with leptin was observed. Each additional WHR-increasing allele in the GPS-WHR was associated with a 0.019 SD or 0.002 WHR-units $\left(p<1.8 \times 10^{-35}\right)$ higher WHR, with the effect being stronger in women than in men $(0.03 \mathrm{SD}$ vs 0.007 $\left.\mathrm{SD}, p_{\text {interaction }}=8.4 \times 10^{-17}\right)(\mathrm{ESM}$ Table 6 , Fig. 2a). The GPS-WHR was not associated with $\mathrm{BF} \%$ or leptin levels (Table 1).

Association with glucose- and insulin-related traits

Two of the $32 \mathrm{BMI}$ loci ( $Q P T C L$ and $R B J$ [also known as $D N A J C 27]$ ) showed evidence of association with glucoserelated traits, whereas none was associated with insulinrelated phenotypes. The BMI-increasing allele at $Q P T C L$ (rs2287019) was associated with lower $2 \mathrm{~h}$ glucose levels, irrespective of adjustment for BMI (without BMI adjustment: -0.108 vs -0.107 SD with BMI adjustment, $p<1.3 \times 10^{-5}$ ). The association of the BMI-increasing allele at $R B J$ (rs713586) with lower $2 \mathrm{~h}$ glucose was more pronounced after adjusting for BMI (without BMI adjustment: -0.060 , $p=1.0 \times 10^{-3}$; with BMI adjustment: $-0.068 \mathrm{SD}, p=1.1 \times 10^{-4}$ ) (Table 2, ESM Table 7). A higher GPS-BMI was associated with higher levels of insulin and proinsulin $(0.011 \mathrm{SD}$ /allele for both, $\left.p<2.3 \times 10^{-3}\right)$. However, these associations were abolished after adjusting for BMI. Interestingly, a higher GPS-BMI was associated with lower $2 \mathrm{~h}$ glucose after, but not before, adjusting for BMI $\left(-0.013 \mathrm{SD} /\right.$ allele, $\left.p=3.6 \times 10^{-4}\right)$ (Table 1, Fig. 1b).

Two of the 14 WHR loci (GRB14 and CPEB4) were associated with glycaemic traits. The WHR-increasing allele at $G R B 14$ (rs10195252) was associated with increased levels of proinsulin, of which the effect size was only slightly 

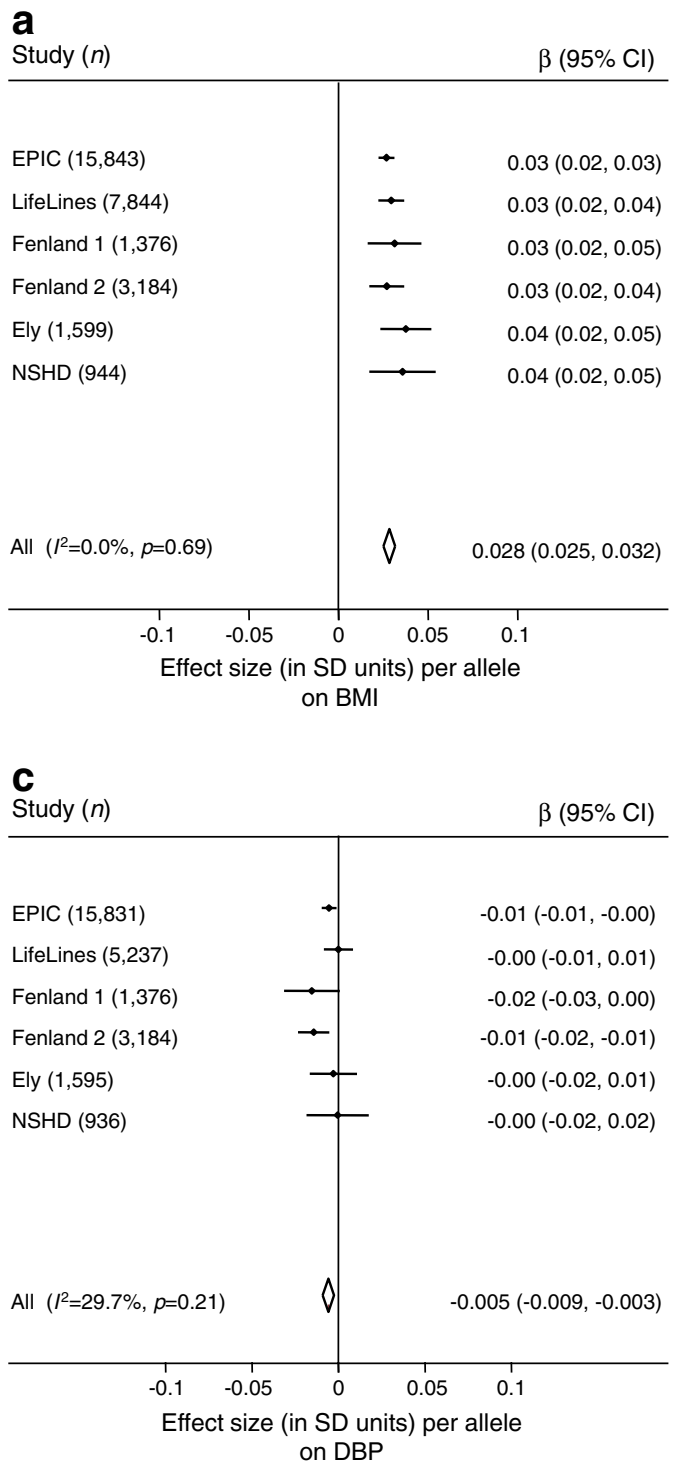

Fig. 1 The combined effects of the BMI-increasing alleles on BMI (a), $2 \mathrm{~h}$ glucose (b) and diastolic BP (DBP) (c) and systolic BP (SBP) (d). The effect was calculated using linear regression in individual studies and after the meta-analysis of $\beta$ values and standard errors using the fixed-effects inverse-variance method. The associations were adjusted for age, sex and BMI (except for the analysis of BMI); associations with

attenuated after adjusting for WHR (0.076 SD and 0.069 SD, respectively, $p<1.2 \times 10^{-5}$ ). Interestingly, the WHR-increasing allele at CPEB4 (rs6861681) was associated with lower fasting glucose, but only after adjusting for WHR $(-0.049$ SD, $p=2.3 \times 10^{-4}$ ) (Table 3, ESM Table 8). The GPS-WHR was not associated with glucose-related traits.

\section{Association with lipid levels}

Four of the 32 BMI loci showed a significant association with blood lipids. Among these, the BMI-increasing alleles of SLC39A8 (rs13107325) and MTCH2 (rs3817334) were b

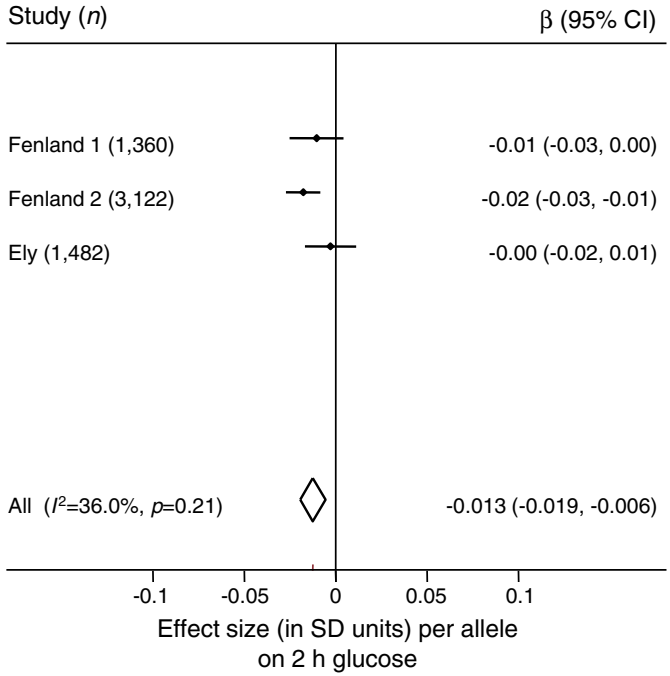

d

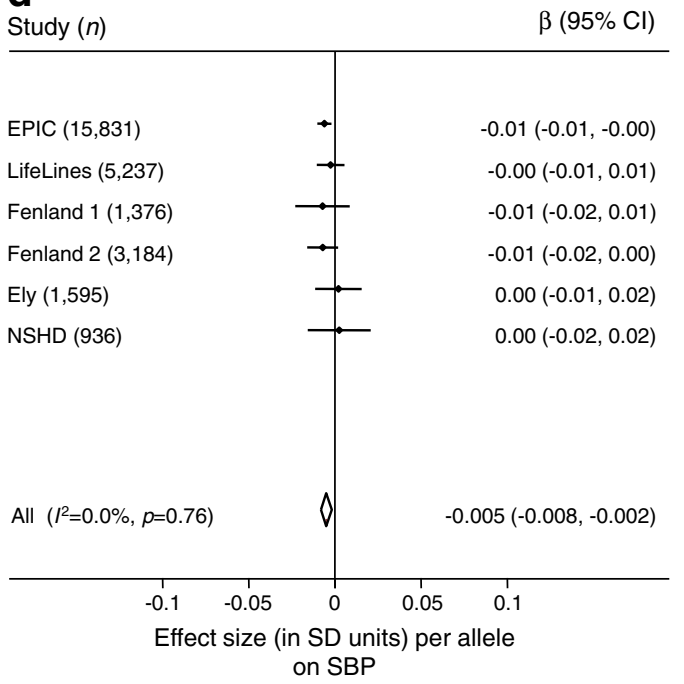

diastolic and systolic BP were additionally adjusted for BP-lowering medication. All diabetic patients were excluded from the analysis. As all traits were inverse normally transformed, $\beta$ values represent the difference in outcome for each additional risk allele under an additive model, expressed in SD units/allele. Risk alleles were defined as the BMI-increasing alleles in the discovery study

associated with lower HDL-cholesterol levels $(-0.079 \mathrm{SD}$ and $-0.046 \mathrm{SD}$ ), and of QPCTL (rs2287019) with higher LDL-cholesterol and total cholesterol levels $(0.042 \mathrm{SD}$ and $0.043 \mathrm{SD}$ ) (all $\left.p<1.0 \times 10^{-4}\right)$. Interestingly, the BMI-increasing allele of FLJ35779 (also known as POC5) (rs2112347) was associated with lower levels of both LDL-cholesterol $(-0.040$ $\mathrm{SD})$ and total cholesterol $(-0.039 \mathrm{SD})\left(p<8.6 \times 10^{-7}\right)$ (Table 2, ESM Table 9). All associations remained significant after adjusting for BMI, without attenuation of effect sizes $\left(p<1.1 \times 10^{-4}\right)$. A higher GPS-BMI was associated with lower levels of HDL-cholesterol and higher levels of triacylglycerol $\left(-0.015 \mathrm{SD}\right.$ and $0.14 \mathrm{SD}$, respectively, $\left.p<1.3 \times 10^{-3}\right)$. 
Table 1 The meta-analysis of the association between the traits and genetic predisposition score for the BMI and WHR loci

\begin{tabular}{|c|c|c|c|c|c|c|c|c|c|c|}
\hline \multirow[t]{3}{*}{ Trait } & \multicolumn{5}{|c|}{ GPS-BMI } & \multicolumn{5}{|c|}{ GPS-WHR } \\
\hline & \multirow[t]{2}{*}{$n$} & \multicolumn{2}{|c|}{ Unadjusted for BMI } & \multicolumn{2}{|c|}{ Adjusted for BMI } & \multirow[t]{2}{*}{$n$} & \multicolumn{2}{|c|}{ Unadjusted for WHR } & \multicolumn{2}{|c|}{ Adjusted for WHR } \\
\hline & & $\beta$ value & $p$ value & $\beta$ value & $p$ value & & $\beta$ value & $p$ value & $\beta$ value & $p$ value \\
\hline \multicolumn{11}{|c|}{ Adiposity-related traits } \\
\hline BMI & 30,790 & 0.028 & $1.1 \times 10^{-61 \dagger}$ & - & - & - & - & - & - & - \\
\hline WHR & - & - & - & - & - & 34,179 & 0.019 & $7.7 \times 10^{-37 \dagger}$ & - & - \\
\hline $\mathrm{BF} \%$ & 16,089 & 0.016 & $1.7 \times 10^{-19 \dagger}$ & & & 17,235 & -0.002 & 0.20 & - & - \\
\hline Leptin & 2,964 & 0.022 & $3.3 \times 10^{-4 \dagger}$ & & & 2,938 & 0.001 & 0.79 & - & - \\
\hline \multicolumn{11}{|c|}{ Glucose- and insulin-related traits } \\
\hline Fasting glucose & 13,976 & 0.003 & 0.19 & -0.005 & 0.03 & 13,945 & -0.001 & 0.85 & -0.003 & 0.36 \\
\hline $2 \mathrm{~h}$ glucose & 5,964 & -0.003 & 0.38 & -0.013 & $3.6 \times 10^{-4 \dagger}$ & 5,938 & 0.000 & 1.00 & -0.005 & 0.38 \\
\hline $\mathrm{HbA}_{1 \mathrm{c}}$ & 22,152 & 0.003 & 0.11 & -0.001 & 0.50 & 23,583 & 0.000 & 0.94 & -0.002 & 0.47 \\
\hline Insulin & 5,929 & 0.011 & $2.3 \times 10^{-3 \dagger}$ & -0.006 & 0.05 & 5,899 & 0.011 & 0.02 & 0.004 & 0.34 \\
\hline Proinsulin & 5,920 & 0.011 & $1.5 \times 10^{-3 \dagger}$ & -0.003 & 0.37 & 5,891 & 0.009 & 0.05 & 0.003 & 0.56 \\
\hline \multicolumn{11}{|l|}{ Blood-lipid traits } \\
\hline HDL-cholesterol & 26,612 & -0.006 & $1.6 \times 10^{-4 \dagger}$ & 0.001 & 0.44 & 29,777 & -0.015 & $7.5 \times 10^{-10 \dagger}$ & -0.011 & $1.3 \times 10^{-4 i}$ \\
\hline LDL-cholesterol & 26,576 & 0.002 & 0.39 & -0.002 & 0.27 & 29,742 & 0.003 & 0.17 & 0.001 & 0.69 \\
\hline Triacylglycerol & 27,229 & 0.006 & $1.3 \times 10^{-3 \dagger}$ & -0.003 & 0.06 & 30,486 & 0.014 & $6.1 \times 10^{-7 \dagger}$ & 0.008 & $2.4 \times 10^{-4 i}$ \\
\hline Total cholesterol & 27,234 & 0.001 & 0.64 & -0.002 & 0.17 & 30,492 & 0.001 & 0.57 & -0.001 & 0.61 \\
\hline DBP & 28,159 & 0.002 & 0.39 & -0.006 & $4.9 \times 10^{-4 \dagger}$ & 31,560 & 0.005 & 0.03 & 0.002 & 0.29 \\
\hline SBP & 28,159 & 0.001 & 0.47 & -0.005 & $1.9 \times 10^{-3 \dagger}$ & 31,560 & 0.004 & 0.10 & 0.002 & 0.49 \\
\hline
\end{tabular}

$\beta$ values are shown as change in SD units per BMI- or WHR-increasing allele. Summary statistics of individual studies were meta-analysed. The analyses were adjusted for sex, age and use of medication (BP and lipids traits). The analyses for the WHR SNPs were additionally adjusted for BMI ${ }^{\dagger}$ Significant association (Bonferroni-corrected significance level, $p<6.7 \times 10^{-3}$ )

DBP, diastolic BP; SBP, systolic BP

However, these associations were abolished after adjusting for BMI (Table 1).

Four WHR loci were significantly associated with lipid traits. The WHR-increasing allele at GRB14 (rs10195252) was associated with lower HDL-cholesterol levels $(-0.033$ $\mathrm{SD}$ ), and those at RSPO3 (rs9491696), VEGFA (rs6905288), and CPEB4 (rs6861681) were associated with higher triacylglycerol levels $(0.029,0.034$, and $0.033 \mathrm{SD}$, respectively $p<1.1 \times 10^{-4}$ ) (Table 3 ). All these associations were abolished after further adjusting for WHR. Amongst the seven WHR-associated loci that were previously reported to have sex-specific effects (17), the WHR-increasing allele at VEGFA was significantly associated with higher LDL-cholesterol levels in women but not in men $(0.045 \mathrm{SD}$ in women $\left[p=1.1 \times 10^{-4}\right]$ and $-0.018 \mathrm{SD}$ in men $[p=0.16]$, $\left.p_{\text {interaction }}=3.3 \times 10^{-4}\right)($ ESM Table 10$)$. The association was only slightly attenuated after further adjustment for WHR (0.040 SD in women $\left[p=6.3 \times 10^{-4}\right]$ and -0.19 in men $[p=0.15])$. A higher GPS-WHR was associated with lower HDL-cholesterol levels and higher triacylglycerol levels without and with further adjusting for WHR $(-0.015$ and 0.014 before and -0.011 and $0.008 \mathrm{SD} /$ allele after the adjustment for
WHR, respectively, $p<2.4 \times 10^{-4}$ ) (Table 3, Fig. 2b, c). We did not observe an association of the GPS-WHR with either LDLcholesterol or total cholesterol levels.

\section{Association with BP}

Of the 32 BMI loci, only the association for the SLC39A8 locus with diastolic BP reached significance, with the BMI-increasing allele being associated with a lower BP $\left(-0.058, p=2.5 \times 10^{-4}\right)$. The association was somewhat more pronounced after adjusting for BMI $(-0.069 \mathrm{SD} /$ allele, $p=5.8 \times 10^{-6}$ ) (Table 2). The GPS-BMI was not associated with BP without adjusting for BMI. After adjustment for BMI, a higher GPS-BMI was associated with lower diastolic and systolic blood BP $(-0.006$ and $-0.005 \mathrm{SD} /$ allele or -0.063 and $-0.09 \mathrm{mmHg} /$ allele, respectively, all $p<1.4 \times 10^{-3}$ ) (Table 1 , Fig. 1c, d, ESM Table 11).

We showed no associations between the individual WHR loci or the GPS-WHR and BP (Tables 1 and 3, ESM Table 12).

There was low $\left(I^{2}<25 \%\right)$ to moderate $\left(I^{2}\right.$ from $25 \%$ to $75 \%)$ between-study heterogeneity for all above-described 
a

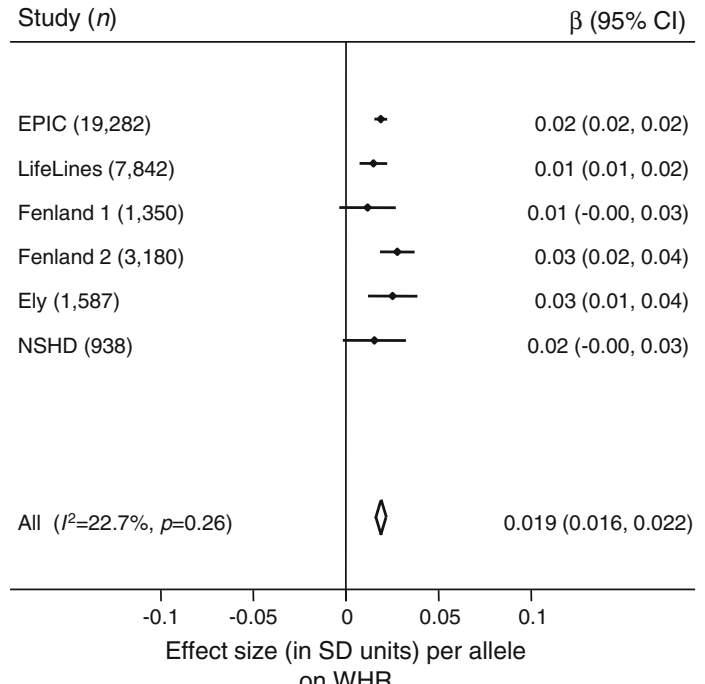

b

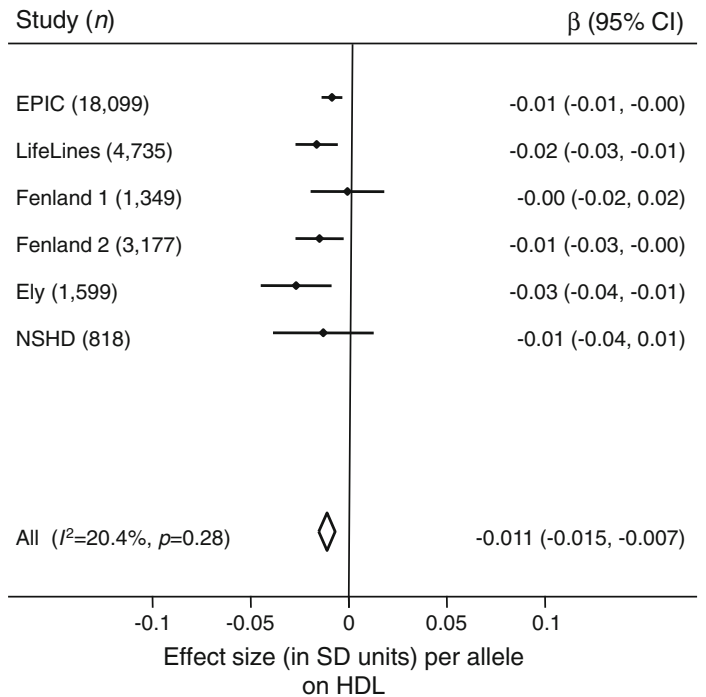

C

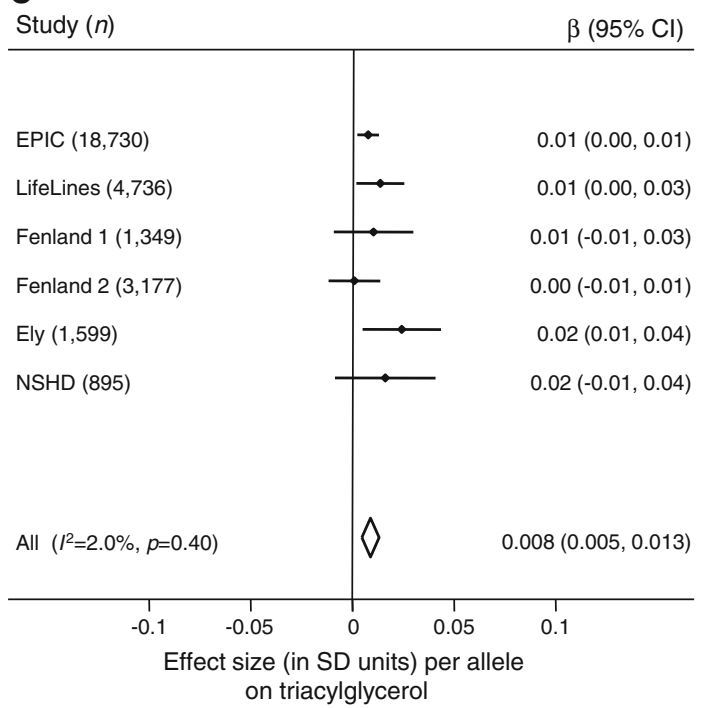

Fig. 2 The combined effects of the WHR-increasing variants on WHR (a), HDL-cholesterol (b) and triacylglycerol (c). The effect was calculated using linear regression in individual studies and after the metaanalysis of $\beta$ values and standard errors using the fixed-effects inversevariance method. Associations were adjusted for age, sex, BMI and WHR (except for the analysis of WHR); associations with lipids were additionally adjusted for blood-lipid-lowering medication. All diabetic patients were excluded from the analysis. As all traits were inverse normally transformed, $\beta$ values represent the difference in outcome for each additional risk allele under an additive model, expressed in SD units/allele. Risk alleles were defined as the WHR-increasing alleles in the discovery study

significant associations (for details see ESM Methods and Results and ESM Tables 13 and 14).

\section{Discussion}

In this report, we examined whether the currently GWASestablished obesity-susceptibility loci also influence metabolic and cardiovascular traits, independent of their effect on obesity-related traits. Our meta-analysis in up to 37,874 European individuals revealed pleiotropic effects for five BMI-, and two WHR-associated loci and demonstrated remarkable diversity in their association patterns with both metabolically 'healthy' and 'unhealthy' obesity phenotypes. Somewhat paradoxically, we further showed that a stronger genetic susceptibility for increased BMI conferred by the 32 loci (GPS-BMI) is associated with lower $2 \mathrm{~h}$ glucose levels and lower systolic and diastolic BP after adjusting for BMI. In contrast, a genetic predisposition for increased WHR (GPS-WHR) predisposes to lower HDL-cholesterol and higher triacylglycerol levels, independent of effects on WHR.

We observed directionally consistent associations for a subset of obesity loci with metabolically 'unhealthy' conditions [7, 8], such as altered levels of insulin-related traits and blood lipids (ESM Table 15). Adjusting the analyses for adiposity traits abolished some of these associations, but revealed several associations that remained significant or stood out after adjusting for BMI or WHR, suggesting pleiotropic effects. A subset of obesity-susceptibility loci remained associated with metabolically 'unhealthy' phenotypes, while for other loci the BMI- and WHR-increasing alleles were associated with metabolically 'healthy' profiles (Table 4).

Several of our pleiotropic associations are consistent with previous reports. First, the associations of QPCTL with $2 \mathrm{~h}$ glucose and blood lipids are in agreement with other studies [27-31]. The QPCTL locus harbours the GIPR gene, identified in GWASs for $2 \mathrm{~h}$ glucose [27-29], which encodes a protein involved in insulin secretion and beta cell function [27-29]. The BMI-associated SNP rs2287019 in QPTCL is in strong LD with coding variants in $\operatorname{GIPR}\left(r^{2}>0.8\right)$ [14]. Of 


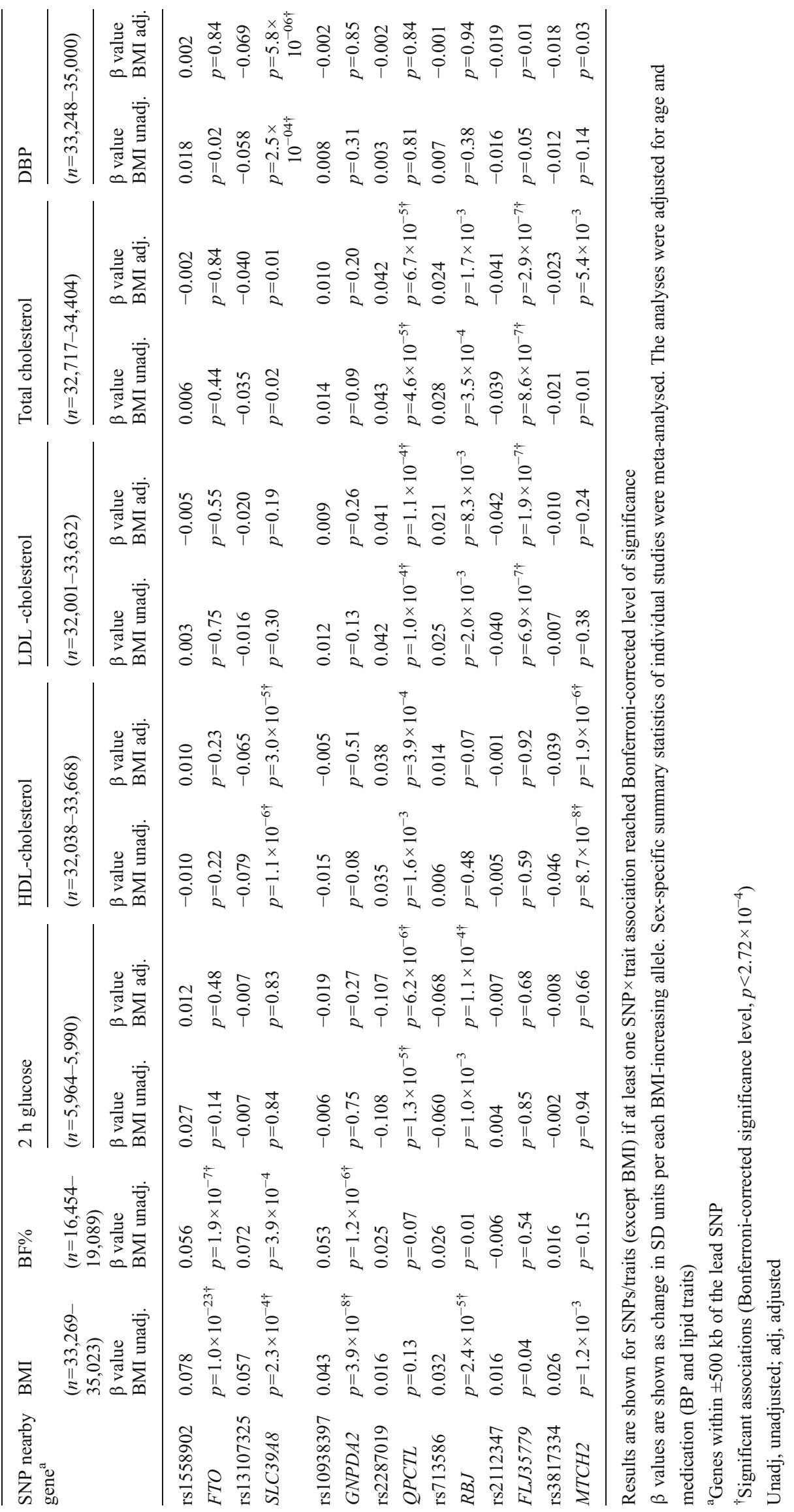




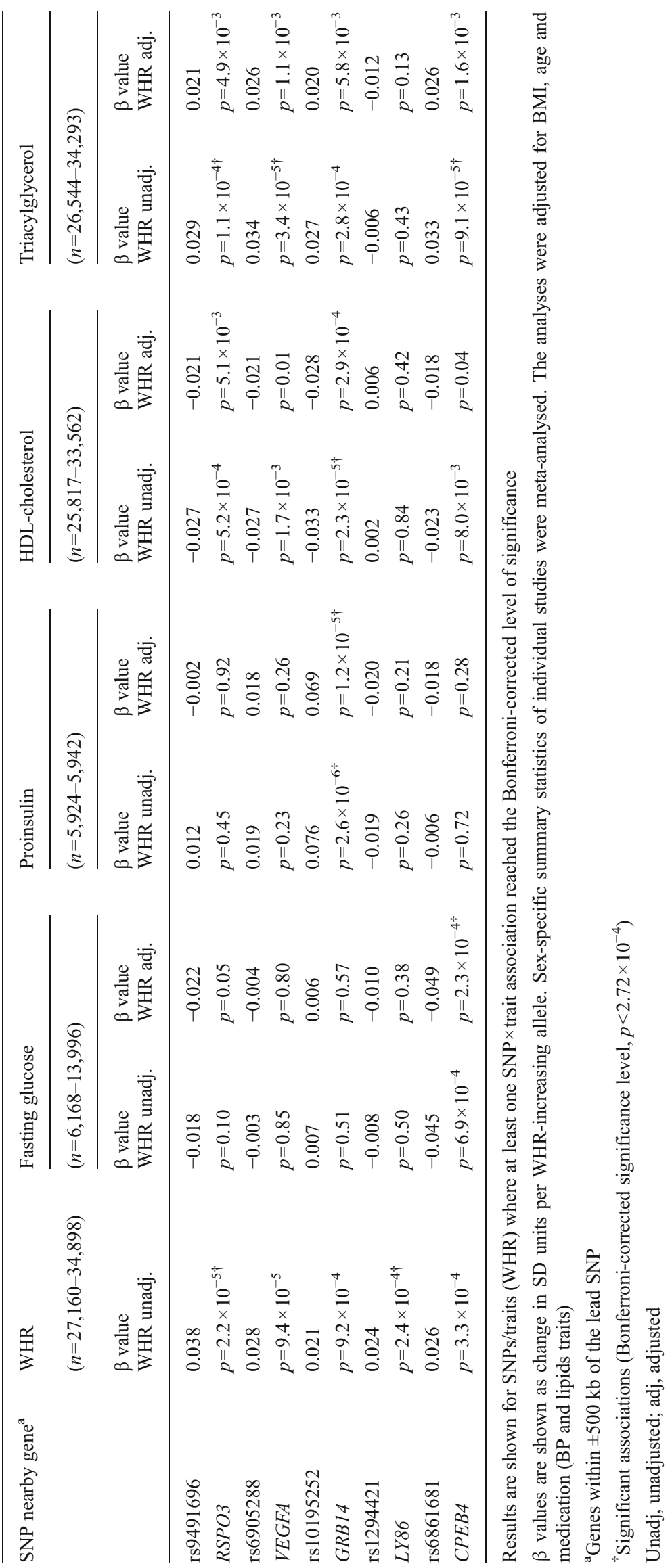


Table 4 Pleiotropic associations of the individual obesity-susceptibility loci and GPSs with metabolic phenotypes

\begin{tabular}{lll}
\hline Phenotypes & $\begin{array}{l}\text { Metabolically } \\
\text { 'unhealthy' associations }\end{array}$ & $\begin{array}{l}\text { Metabolically } \\
\text { 'healthy' associations }\end{array}$ \\
\hline $\begin{array}{l}\text { Glucose- and insulin-related traits } \\
\text { Fasting glucose }\end{array}$ & CPEB4 \\
2 h glucose & & QPTCL, RBJ, \\
& & \\
Proinsulin & GRB14 & \\
Blood-lipid traits & & \\
HDL-cholesterol & SLC39A8, MTCH2, \\
& $G R B 14$, GPS-WHR & FLJ35779 \\
LDL-cholesterol & QPTCL & \\
Triacylglycerol & GPS-WHR & FLJ35779 \\
Total cholesterol & QPTCL & \\
BP traits & & SLC39A8, GPS-BMI \\
DBP & & GPS-BMI \\
SBP & &
\end{tabular}

DBP, diastolic BP; SBP, systolic BP

particular interest is the pattern of association, with the BMIincreasing allele being associated with lower $2 \mathrm{~h}$ glucose, as reported previously $[27,30]$, which is opposite to what would be expected based on known epidemiological correlations. Yet, consistent with epidemiological correlations, the adiposity-increasing allele was associated with higher levels of LDL-cholesterol and total cholesterol. Second, associations between the BMI-increasing allele of rs13107325 in SLC39A8 with both metabolically 'unhealthy' (lower HDL-cholesterol) and metabolically 'healthy' (lower diastolic BP) phenotypes confirm findings from GWASs for BP and blood lipids [32-34]. The BMI-associated variant rs 13107325 is a non-synonymous SNP located in exon 7 [34] of the SLC39A8 gene, which encodes a zinc transporter involved in zinc transportation into the cell at the onset of inflammation $[35,36]$. As indicated recently, zinc deficiency is also associated with lower BP [37]. Third, the associations of the WHR-increasing allele at GRB14 with metabolically 'unhealthy' phenotypes (higher proinsulin and lower HDL-cholesterol) have been confirmed in the most recent meta-analyses for glycaemic traits (the Meta-Analyses of Glucose and Insulin-related traits Consortium [MAGIC]) [30] and type 2 diabetes (the DIAbetes Genetics Replication And Meta-analysis [DIAGRAM] consortium) [31], and in a GWAS for HDL-cholesterol [38]. Recent analysis of expression quantitative trait locus (eQTL) data implicates GRB14 as a potential causal gene at the locus, expressed in the liver, skeletal muscle and adipose tissue [39].

Furthermore, some observed associations suggest novel pleiotropic effects. For example, we detected the relationship of rs2112347 near FLJ35779 and a more favourable lipid profile. This variant is located approximately $400 \mathrm{~kb}$ from $H M G C R 3$, involved in lipid metabolism and shown to be associated with total cholesterol [32]. The biological mechanisms by which the remaining obesity-susceptibility loci might affect glucose (RBJ, CPEB4) and lipid metabolism $(M T C H 2)$ are less well understood [40-42] and require further investigation.

We show that a greater susceptibility to increased WHR, as assessed by the GPS, is associated with a metabolically unfavourable lipid profile, even after adjustment for adiposity. In contrast, the associations of a higher GPS-BMI with metabolically unhealthy levels of insulin- or lipid-related traits were abolished after adjusting for BMI. Similar observations of the associations between the obesity risk alleles and insulin resistance, mediated by BMI, were reported previously [43, 44]. Even though trends of associations are consistent across studies, associations of FTO with a number of metabolic traits in a BMI-dependent manner do not reach our significance threshold, which accounts for multiple testing, whereas previous studies used nominal levels [44]. In contrast, a higher GPS-BMI was associated with lower levels of $2 \mathrm{~h}$ glucose and BP after adjusting for BMI. These results support recent finding of the association of the BMIincreasing alleles collectively with increased beta cell capacity [45]. Particularly intriguing is the novel 'pleiotropic' association of a higher GPS-BMI with decreased BP. While $60-70 \%$ of hypertension in adults may be directly attributable to adiposity $[4,46]$, some discrepancies in the BPadiposity relationship have also been described. For example, Pima Indians, who have the highest prevalence of reported obesity in the world, have a low prevalence of hypertension [47]. To summarise, the genetic pleiotropic associations may in part explain why some obese individuals remain metabolically healthy despite having excessive accumulation of body fat [48-53]. This phenomenon is described in the current literature as 'healthy obesity' and, to date, little is known about the factors that protect obese individuals from metabolic disturbances [54].

Our results provide evidence for pleiotropic effects of obesity-susceptibility loci on metabolic traits, supporting the hypothesis on the shared nature of genetic susceptibility loci in related complex diseases [10]. A recent analysis of the loci identified in GWASs for common diseases and quantitative traits showed pleiotropic effects for almost $17 \%$ of analysed genes and for $4.6 \%$ of SNPs [55]. These and our findings indicate that pleiotropy is more common in complex diseases than previously anticipated, and suggest that many proteins play different roles in independent biological pathways. Possible explanations for pleiotropic effects are that positive genetic correlations between phenotypic traits (i.e. affecting the traits in the same direction) occur if they share common biological pathways, while negative genetic 
correlations might be 'evolutionarily' beneficial when a genetic mutation favourable to one trait can be harmful for another trait [56]. Interestingly, we observed both types of genetic correlation in our study. Among the detected pleiotropic effects of both single-SNP and GPS analyses, $50 \%$ of the significant associations were directionally consistent with a BMI- or WHR-increasing allele or a higher GPS associated with metabolically 'unhealthy' phenotypes, while the other half showed unexpected relationships with metabolically 'healthy' phenotypes (Table 4). In addition, these pleiotropic association signatures extend the genetic associations of the obesity-susceptibility loci to related metabolic traits and diseases and provide a more comprehensive and integrated overview of the potential physiological mechanisms of the loci. These insights may eventually help to prioritise genes for functional follow-up and to guide physiologists in designing their functional studies in experimental settings.

There are some limitations to our study. First, only populations from northern European ancestry and obesitysusceptibility loci identified in European ancestry populations were included in our analyses. Cross-ancestry analyses will be required to investigate whether the observed pleiotropic associations in European ancestry populations generalise to other ancestries. Furthermore, the cross-sectional design and the exclusion of diabetic patients are further potential limitations of our study. Future investigations in large prospective studies can be informative in disentangling whether the identified loci affect other metabolic traits through either first influence obesity risk, or whether the association is truly independent of BMI and WHR.

In conclusion, our results provide evidence that obesitysusceptibility loci have pleiotropic effects on metabolic traits, independent of adiposity. These findings emphasise the importance of detailed physiological characterisation of novel obesity-susceptibility loci to gain new insight into the complex pathogenesis of metabolically related disorders. Importantly, our findings highlight that the effect alleles that predispose to obesity might also predispose to, or protect from, other metabolic disorders.

Acknowledgements The authors would like to thank the study teams who collected the data used in these analyses. We also acknowledge the volunteers, who gave their time to take part in the individual studies.

The authors thank their colleagues from University of Groningen, University Medical Center Groningen, Groningen: B. Alizadeh, A. Boesjes, N. Festen, I. Nolte, L. Franke and M. Valimohammadi for their help in creating the GWAS database, and R. Bieringa, C. Döling, M. Elderson, J. Keers, E. Klaver, R. Oostergo, S. Scholtens, R. Visser and J. Vonk for their work related to data collection and validation. The authors are grateful to the study participants, the staff from the LifeLines Cohort Study and Medical Biobank Northern Netherlands, and the participating general practitioners and pharmacists.

Funding The EPIC-Norfolk Study was supported by Cancer Research UK and the MRC (MC U106179471 \& G1000143). The
LifeLines Cohort Study and the generation and management of GWAS genotype data for the LifeLines Cohort Study is supported by the Netherlands Organization of Scientific Research NWO (grant 175.010.2007.006), the Economic Structure Enhancing Fund (FES) of the Dutch government, the Ministry of Economic Affairs, the Ministry of Education, Culture and Science, the Ministry for Health, Welfare and Sports, the Northern Netherlands Collaboration of Provinces (SNN), the Province of Groningen, University Medical Center Groningen, the University of Groningen, the Dutch Kidney Foundation and the Dutch Diabetes Research Foundation. Statistical analyses were carried out on the Genetic Cluster Computer (www.geneticcluster.org), which is financially supported by the Netherlands Scientific Organization (NWO 480-05-003) along with a supplement from the Dutch Brain Foundation and the VU University Amsterdam. The Fenland study was supported by the Wellcome Trust, the MRC, the Support for Science Funding programme and CamStrad (MC U106179471). The MRC Ely Study was supported by the MRC and the Wellcome Trust (MC_U106179471). The MRC NSHD was supported by the MRC (U120063239 \& U123092720). J. V. van Vliet-Ostaptchouk is supported by a Rubicon grant from the Netherlands Organization for Scientific Research (NWO file nr 825.10.035) and the Netherlands Consortium for Healthy Ageing (NCHA) (NCHA NGI Grant 050-060-810). The research leading to these results has received funding from the European Union Seventh Framework Programme (FP7/2007-2013) under grant agreement number 261433.

Contribution statement JVvV-O contributed to the conception of the study, performed the statistical analysis, analysed and interpreted the data and wrote the manuscript. RJFL conceived and designed the research, analysed and interpreted the data and made critical revisions of the manuscript for important intellectual content. $\mathrm{MdH}$ and $\mathrm{HS}$ analysed and interpreted the data and made critical revisions of the manuscript for important intellectual content. PJvdM provided help on statistical analysis and reviewed the manuscript. JL, JHZ, MMvdK and MB acquired data, contributed to the clinical characterisation of samples, sample preparation and QC, biochemical profiles and database handling, and reviewed the manuscript. KKO, AW, RH, DK, BHRW, KTK and NJW acquired data, provided study materials and reviewed the manuscript. All authors read and approved the final manuscript. JVVV-O and RJFL are the guarantors of this work and, as such, had full access to all the data in the study and take responsibility for the integrity of the data and the accuracy of the data analysis.

Duality of interest The authors declare that there is no duality of interest associated with this manuscript.

\section{References}

1. Feller S, Boeing H, Pischon T (2010) Body mass index, waist circumference, and the risk of type 2 diabetes mellitus: implications for routine clinical practice. Dtsch Arztebl Int 107:470-476

2. Canoy D, Boekholdt SM, Wareham N et al (2007) Body fat distribution and risk of coronary heart disease in men and women in the European Prospective Investigation Into Cancer and Nutrition in Norfolk cohort: a population-based prospective study. Circulation 116:2933-2943

3. Pischon T, Nothlings U, Boeing H (2008) Obesity and cancer. Proc Nutr Soc 67:128-145

4. Mokdad AH, Ford ES, Bowman BA et al (2003) Prevalence of obesity, diabetes, and obesity-related health risk factors, 2001. JAMA 289:76-79

5. Pischon T, Boeing H, Hoffmann K et al (2008) General and abdominal adiposity and risk of death in Europe. N Engl J Med 359:2105-2120 
6. Prospective Studies C, Whitlock G, Lewington S et al (2009) Body-mass index and cause-specific mortality in 900000 adults: collaborative analyses of 57 prospective studies. Lancet 373:10831096

7. Despres JP, Lemieux I (2006) Abdominal obesity and metabolic syndrome. Nature 444:881-887

8. Kahn BB, Flier JS (2000) Obesity and insulin resistance. J Clin Invest 106:473-481

9. Wagner GP, Zhang J (2011) The pleiotropic structure of the genotype-phenotype map: the evolvability of complex organisms. Nat Rev Genet 12:204-213

10. Becker KG (2004) The common variants/multiple disease hypothesis of common complex genetic disorders. Med Hypotheses 62:309-317

11. Frayling TM, Timpson NJ, Weedon MN et al (2007) A common variant in the FTO gene is associated with body mass index and predisposes to childhood and adult obesity. Science 316:889-894

12. Scuteri A, Sanna S, Chen WM et al (2007) Genome-wide association scan shows genetic variants in the FTO gene are associated with obesity-related traits. PLoS Genet 3:e115

13. Loos RJ, Lindgren CM, Li S et al (2008) Common variants near MC4R are associated with fat mass, weight and risk of obesity. Nat Genet 40:768-775

14. Speliotes EK, Willer CJ, Berndt SI et al (2010) Association analyses of 249,796 individuals reveal 18 new loci associated with body mass index. Nat Genet 42:937-948

15. Thorleifsson G, Walters GB, Gudbjartsson DF et al (2009) Genome-wide association yields new sequence variants at seven loci that associate with measures of obesity. Nat Genet 41:18-24

16. Willer CJ, Speliotes EK, Loos RJ et al (2009) Six new loci associated with body mass index highlight a neuronal influence on body weight regulation. Nat Genet 41:25-34

17. Heid IM, Jackson AU, Randall JC et al (2010) Meta-analysis identifies 13 new loci associated with waist-hip ratio and reveals sexual dimorphism in the genetic basis of fat distribution. Nat Genet 42:949-960

18. Lindgren CM, Heid IM, Randall JC et al (2009) Genome-wide association scan meta-analysis identifies three loci influencing adiposity and fat distribution. PLoS Genet 5:e1000508

19. Day N, Oakes S, Luben R et al (1999) EPIC-Norfolk: study design and characteristics of the cohort. European Prospective Investigation of Cancer. Br J Cancer 80(Suppl 1):95-103

20. Stolk RP, Rosmalen JG, Postma DS et al (2008) Universal risk factors for multifactorial diseases: LifeLines: a three-generation population-based study. Eur J Epidemiol 23:67-74

21. Rolfe Ede L, Loos RJ, Druet C et al (2010) Association between birth weight and visceral fat in adults. Am J Clin Nutr 92:347-352

22. Watkinson C, van Sluijs EM, Sutton S, Marteau T, Griffin SJ (2010) Randomised controlled trial of the effects of physical activity feedback on awareness and behaviour in UK adults: the FAB study protocol [ISRCTN92551397]. BMC Publ Health $10: 144$

23. Ekelund U, Brage S, Franks PW, Hennings S, Emms S, Wareham NJ (2005) Physical activity energy expenditure predicts progression toward the metabolic syndrome independently of aerobic fitness in middle-aged healthy Caucasians: the Medical Research Council Ely Study. Diabetes Care 28:1195-1200

24. Kuh D, Pierce M, Adams J et al (2011) Cohort profile: updating the cohort profile for the MRC National Survey of Health and Development: a new clinic-based data collection for ageing research. Int J Epidemiol 40:e1-e9

25. Wadsworth M, Kuh D, Richards M, Hardy R (2006) Cohort Profile: The 1946 National Birth Cohort (MRC National Survey of Health and Development). Int J Epidemiol 35:49-54
26. Willer CJ, Li Y, Abecasis GR (2010) METAL: fast and efficient meta-analysis of genomewide association scans. Bioinformatics 26:2190-2191

27. Saxena R, Hivert MF, Langenberg C et al (2010) Genetic variation in GIPR influences the glucose and insulin responses to an oral glucose challenge. Nat Genet 42:142-148

28. Ingelsson E, Langenberg C, Hivert MF et al (2010) Detailed physiologic characterization reveals diverse mechanisms for novel genetic loci regulating glucose and insulin metabolism in humans. Diabetes 59:1266-1275

29. Lyssenko V, Eliasson L, Kotova O et al (2011) Pleiotropic effects of GIP on islet function involve osteopontin. Diabetes 60:2424-2433

30. Scott RA, Lagou V, Welch RP et al (2012) Large-scale association analyses identify new loci influencing glycemic traits and provide insight into the underlying biological pathways. Nat Genet 44:991-1005

31. the DGR, Meta-analysis C, Morris AP et al (2012) Largescale association analysis provides insights into the genetic architecture and pathophysiology of type 2 diabetes. Nat Genet 44:981-990

32. Teslovich TM, Musunuru K, Smith AV et al (2010) Biological, clinical and population relevance of 95 loci for blood lipids. Nature 466:707-713

33. Waterworth DM, Ricketts SL, Song K et al (2010) Genetic variants influencing circulating lipid levels and risk of coronary artery disease. Arterioscler Thromb Vasc Biol 30:2264-2276

34. International Consortium for Blood Pressure Genome-Wide Association S, Ehret GB, Munroe PB et al (2011) Genetic variants in novel pathways influence blood pressure and cardiovascular disease risk. Nature 478:103-109

35. Besecker BY, Exline MC, Hollyfield J et al (2011) A comparison of zinc metabolism, inflammation, and disease severity in critically ill infected and noninfected adults early after intensive care unit admission. Am J Clin Nutr 93:1356-1364

36. Liuzzi JP, Cousins RJ (2004) Mammalian zinc transporters. Annu Rev Nutr 24:151-172

37. Tubek S (2007) Role of zinc in regulation of arterial blood pressure and in the etiopathogenesis of arterial hypertension. Biol Trace Elem Res 117:39-51

38. Ridker PM, Pare G, Parker AN, Zee RY, Miletich JP, Chasman DI (2009) Polymorphism in the CETP gene region, HDL cholesterol, and risk of future myocardial infarction: genomewide analysis among 18245 initially healthy women from the Women's Genome Health Study. Circ Cardiovasc Genet 2:26-33

39. Goenaga D, Hampe C, Carre N et al (2009) Molecular determinants of Grb14-mediated inhibition of insulin signaling. Mol Endocrinol 23:1043-1051

40. Kulyte A, Ryden M, Mejhert N et al (2011) MTCH2 in human white adipose tissue and obesity. J Clin Endocrinol Metab 96:E1661-E1665

41. Okada S, Matsuda M, Anafi M, Pawson T, Pessin JE (1998) Insulin regulates the dynamic balance between Ras and Rap1 signaling by coordinating the assembly states of the Grb2-SOS and CrkII-C3G complexes. EMBO J 17:2554-2565

42. Ortiz-Zapater E, Pineda D, Martinez-Bosch N et al (2012) Key contribution of CPEB4-mediated translational control to cancer progression. Nat Med 18:83-90

43. Robiou-du-Pont S, Bonnefond A, Yengo L et al (2012) Contribution of 24 obesity-associated genetic variants to insulin resistance, pancreatic beta-cell function and type 2 diabetes risk in the French population. Int J Obes (Lond). doi:10.1038/ijo.2012.175

44. Freathy RM, Timpson NJ, Lawlor DA et al (2008) Common variation in the FTO gene alters diabetes-related metabolic traits to the extent expected given its effect on BMI. Diabetes 57:1419-1426 
45. Burgdorf KS, Gjesing AP, Grarup N et al (2011) Association studies of novel obesity-related gene variants with quantitative metabolic phenotypes in a population-based sample of 6,039 Danish individuals. Diabetologia 55:105-113

46. Must A, Spadano J, Coakley EH, Field AE, Colditz G, Dietz WH (1999) The disease burden associated with overweight and obesity. JAMA 282:1523-1529

47. Kotchen TA (2010) Obesity-related hypertension: epidemiology, pathophysiology, and clinical management. Am J Hypertens 23:1170-1178

48. Geetha L, Deepa M, Anjana RM, Mohan V (2011) Prevalence and clinical profile of metabolic obesity and phenotypic obesity in Asian Indians. J Diabetes Sci Technol 5:439-446

49. Kantartzis K, Machann J, Schick F et al (2011) Effects of a lifestyle intervention in metabolically benign and malign obesity. Diabetologia 54:864-868

50. Pajunen P, Kotronen A, Korpi-Hyovalti E et al (2011) Metabolically healthy and unhealthy obesity phenotypes in the general population: the FIN-D2D Survey. BMC Publ Health 11:754
51. Stefan N, Kantartzis K, Machann J et al (2008) Identification and characterization of metabolically benign obesity in humans. Arch Intern Med 168:1609-1616

52. Velho S, Paccaud F, Waeber G, Vollenweider P, Marques-Vidal P (2010) Metabolically healthy obesity: different prevalences using different criteria. Eur J Clin Nutr 64:1043-1051

53. Wildman RP, Muntner P, Reynolds K et al (2008) The obese without cardiometabolic risk factor clustering and the normal weight with cardiometabolic risk factor clustering: prevalence and correlates of 2 phenotypes among the US population (NHANES 1999-2004). Arch Intern Med 168:1617-1624

54. Pataky Z, Bobbioni-Harsch E, Golay A (2010) Open questions about metabolically normal obesity. Int J Obes 34(Suppl 2):S18-S23

55. Sivakumaran S, Agakov F, Theodoratou E et al (2011) Abundant pleiotropy in human complex diseases and traits. Am J Hum Genet 89:607-618

56. Mackay TF, Stone EA, Ayroles JF (2009) The genetics of quantitative traits: challenges and prospects. Nat Rev Genet 10:565-577 